\title{
Cytokine correlates of clinical response patterns to infliximab treatment of ankylosing spondylitis
}

\author{
M A Stone, U Payne, C Pacheco-Tena, R D Inman
}

Ann Rheum Dis 2004;63:84-87. doi: 10.1136/ard.2003.006916

Objective: To identify clinical and immunological markers of response to treatment with infliximab in ankylosing spondylitis (AS).

Methods: Baseline and sequential cytokine levels (IL1, TNF $\alpha$, IFN $\gamma$, TGF $\beta$ and IL10) were examined after 52 weeks of infliximab treatment $5 \mathrm{mg} / \mathrm{kg}$ in 22 patients.

Results: At week 52, 18 patients were responders and four non-responders according to ASAS group criteria. Clinical measures of disease activity between the two groups at baseline were similar, apart from a trend towards longer disease duration in non-responders $(p=0.08)$. Baseline CRP and TNF $\alpha$ levels were higher in responders than nonresponders $(p<0.01$ and $p<0.006$, respectively). The two groups had similar baseline cytokine levels, apart from TNF $\alpha$. Baseline CRP levels did not correlate significantly with baseline cytokine levels in responders, but a strong correlation was noted between baseline CRP and ILI, IFN $\gamma$, and ILIO in non-responders. Apart from an early rise in TGF $\beta$ and a decrease in IL10 in responders after the first infusion, sequential cytokine analysis for the first six months of treatment was not related to clinical disease activity measures.

Conclusion: Although sequential cytokine analysis does not appear to be informative, baseline CRP and TNF $\alpha$ levels are useful markers of clinical response patterns in patients with AS treated with infliximab.
C vincing evidence from several short term randomised controlled trials indicates that treatment of ankylosing spondylitis (AS) with biological agents (infliximab and etanercept) is safe and efficacious. ${ }^{1-3}$ However, a subset of patients fails to respond or does not sustain initial responses. It is thus imperative to define variability in clinical response in order to guide the optimal use of this agent. To date the effect of infliximab on cytokine networks in AS has not been fully defined, although two recent well designed studies have examined the issue. ${ }^{45}$

Neither of these studies, however, aimed at identifying the cytokine profile of patients stratified by response to treatment. Because infliximab is a biological agent that specifically targets tumour necrosis factor (TNF) it seems logical to look for differences in the cytokine profile of patients treated with this agent to identify those patients most likely to respond. In this study we attempt to identify clinical and immunological markers of response to treatment with infliximab in AS. We examined sequential cytokine levels (interleukin (IL) $1, \mathrm{TNF} \alpha$, interferon $\gamma$ (IFN $\gamma$ ), transforming growth factor $\beta$ (TGF $\beta$ ), and IL10) after 52 weeks of infliximab treatment in 22 patients.

\section{METHODS}

\section{Patient ascertainment}

Twenty two consecutive patients with AS according to the modified New York criteria ${ }^{6}$ who had active disease, and for whom non-steroidal anti-inflammatory drug (NSAID) treatment had failed, received infliximab (Remicade; manufactured by Centocor, Malvern, PA and distributed by Schering Plough, Canada Inc) intravenously at baseline, two weeks, six weeks, and every eight weeks thereafter for one year, at a dose of $5 \mathrm{mg} / \mathrm{kg}$. Twenty one of the 22 patients have been described in detail previously. ${ }^{7}$

The institutional ethics committee reviewed the protocol and all patients signed informed consent.

\section{Laboratory methods}

Serial cytokine (ILl, IL10, TGF $\beta$, TNF $\alpha$, and IFN $\gamma$ ) determinations were measured on peripheral blood mononuclear cells (PBMC) at baseline, weeks 2, 6, 14, and 22. The blood sample was drawn just before the next infusion of infliximab was initiated. PBMC were isolated by Ficoll Hypaque (Pharmacia, Uppsala, Sweden) centrifugation, and resuspended in RPMI. $0.2 \mathrm{ml}$ of the cells was added to a 96 well flat bottomed plate (Costar) at a final concentration of $10^{5}$ cells per well. The cells were cultured for 48 hours with or without $1 \mu \mathrm{g} / \mathrm{ml}$ phytohaemagglutinin (Gibco BRL). The supernatants were collected and frozen at $-60^{\circ} \mathrm{C}$.

The respective cytokines were measured by a quantitative sandwich enzyme immunoassay kit (R\&D Systems Inc, Minneapolis, MN). All samples were batched and assayed in a single run to minimise interassay variability.

A polyclonal antibody specific for the cytokine to be tested was precoated onto the microplate. Standards and samples were pipetted into the wells and the test cytokine was bound by the immobilised antibody. After washing, the enzyme linked polyclonal antibody against the cytokine was added to the wells. After another wash, a substrate solution was added to the wells and the colorimetric reaction read in an enzyme linked immunosorbent assay (ELISA) reader at $450 \mathrm{~nm}$. Serum levels of cytokines were below the lowest standard within the respective ELISA so supernatants on the stimulated PBMC were the test variables.

$C$ reactive protein (CRP) and erythrocyte sedimentation rate (ESR) were measured by standard laboratory methods.

\footnotetext{
Abbreviations: ANOVA, analysis of variance; AS, ankylosing spondylitis; ASAS, AS Assessment Study; BASDAI, Bath AS Disease Activity Index; BASFI, Bath AS Spondylitis Index; CRP, C reactive protein; DMARD, disease modifying antirheumatic drug; ELISA, enzyme linked immunosorbent assay; ESR, erythrocyte sedimentation rate; IFN $\gamma$, interferon $\gamma$; IL, interleukin; NSAID, non-steroidal anti-inflammatory drug; PBMC, peripheral blood mononuclear cells; SpA, spondyloarthropathy; TGF $\beta$, transforming growth factor $\beta$; TNF $\alpha$, tumour necrosis factor $\alpha$; VAS, visual analogue scale
} 


\section{Primary outcome measures}

The following measures were obtained at baseline and each follow up visit: patient global assessment (five point Likert scale: $0=$ best, $4=$ worst), patient pain (visual analogue scale (VAS): $0=$ none, $100=$ maximum), Bath AS Functional Index (BASFI) score $(0=$ none, $100=$ worst $),{ }^{8}$ early morning stiffness (calculated as a mean of the following two scales $(a)$ VAS: $0=$ no stiffness, $100=$ maximum average early morning stiffness in the preceding week and (b) VAS $100 \mathrm{~mm}$ categorical scale of stiffness: 0-120 minutes in 30 minute increments), and physician global assessment (four point Likert scale: $0=$ best, $3=$ worst).

Response to treatment at week 52 was defined by the AS Assessment Study (ASAS) 20 criteria. ${ }^{9}$ ASAS response criteria are defined as follows: $\geqslant 20 \%$ improvement and an absolute improvement of $\geqslant 10$ units in three of four of the following domains: inflammation (early morning stiffness), function (BASFI), patient perception of pain (patient pain VAS), and patient global assessment, with no worsening in the fourth domain. The cohort was divided into two groups according to these criteria at week 52. A cut off point of $50 \%$ relative improvement for the ASAS response criteria (that is, ASAS 50 response) was used to stratify the response of the cohort to treatment.

\section{Statistical analysis}

The intergroup comparisons of patients' demographics, disease activity measures, and cytokine levels at baseline were made with independent samples $t$ test. Repeated measures analysis of variance (ANOVA) approach was used to determine whether there were any significant differences between the two groups of patients over the study period.

$\chi^{2}$ Tests (Fisher's exact test where the assumptions for a $\chi^{2}$ test were not met) were computed to compare change in clinical disease activity (Bath AS Disease Activity Index (BASDAI) or BASFI, ESR, CRP) and candidate cytokines (TGF $\beta$, IFN $\gamma$, ILl, ILl0, TNF $\alpha$ ) in the two groups, after two weeks' treatment (that is, after the first infusion) and after six weeks' treatment (that is, after induction of treatment). A $\mathrm{p}$ value $<0.05$ on a two tailed $t$ test was considered significant.

Spearman's rank correlation coefficient was used to assess the relationship between clinical (BASDAI) or immunological (CRP) measures of disease activity and the candidate cytokines. A correlation coefficient $\left(r_{\mathrm{s}}\right)<0.5$ was considered a poor correlation, $r_{\mathrm{s}}=0.5-0.7$ a moderate correlation, and $r_{\mathrm{s}}>0.7$ was considered a strong correlation. Statistical analysis by intention to treat was performed using SAS (SAS institute Cary, NC version 8.02).

\section{RESULTS}

\section{Clinical parameters}

At week 52 there were 18 responders and four nonresponders by ASAS 20 response criteria. Both responders and non-responders were of similar age (39.7 (8.5) and 40.3 (6.5) years, respectively). Responders had a shorter mean disease duration (9 (7.2) years) than non-responders (16 (5.7) years), which approached statistical significance $(p=0.08)$. There was no difference in the use of either NSAIDs or disease modifying antirheumatic drugs (DMARDs) between the two groups. Furthermore, there were no discriminating clinical measures of disease activity or function between responders and non-responders at baseline (table 1). There was a difference in inflammatory measures of disease activity (CRP) between the two groups at baseline, with responders having higher CRP levels than nonresponders $(\mathrm{p}<0.01)$ (fig $1 \mathrm{~A})$.

There was a poor correlation between baseline CRP and BASDAI $\left(r_{\mathrm{s}}=0.36\right)$ for the whole cohort (of 22 patients),
Table 1 Clinical and demographic characteristics of patients stratified by ASAS 20 response to treatment*

\begin{tabular}{llll}
\hline & $\begin{array}{l}\text { Responders } \\
(\mathbf{n}=18)\end{array}$ & $\begin{array}{l}\text { Non-responders } \\
(\mathbf{n}=4)\end{array}$ & $\mathbf{p}$ Value \\
\hline Sex (M:F) & $15: 3$ & $3: 1$ & 0.78 \\
Age (years) & $39.7(8.5)$ & $40.3(6.5)$ & 0.9 \\
Disease duration (years) & $9(7.2)$ & $16(5.7)$ & 0.08 \\
NSAID treatment & 14 & 3 & 0.14 \\
DMARD treatment & 7 & 2 & 0.7 \\
BASDAl & $5.8(1.15)$ & $5.6(2.2)$ & 0.39 \\
BASFI & $6(2.4)$ & $6(2.2)$ & 0.9 \\
CRP (mg/l) & $307.2(359)$ & $62.5(49.9)$ & 0.01 \\
TNF $\alpha(\mathrm{pg} / \mathrm{ml})$ & $4797.8(2793)$ & $2607(546)$ & 0.006 \\
\hline
\end{tabular}

Results are shown as mean (SD)

*Groups were stratified by ASAS 20 response criteria, see "Methods" for a detailed description of ASAS.

BASDAI, Bath Ankylosing Spondylitis Disease Activity Index; BASFI, Bath

Ankylosing Spondylitis Functional Index.
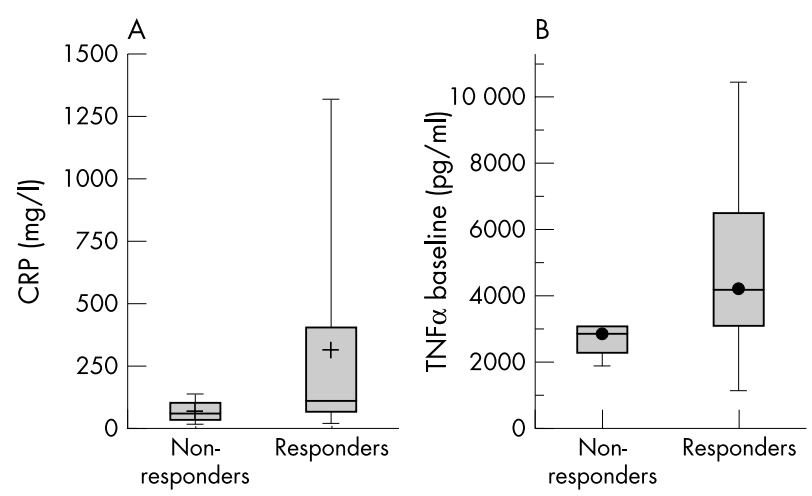

GroupASAS

Figure 1 (A) Comparison of CRP levels by ELISA between responders and non-responders to infliximab in patients with AS. Responders were defined as those patients who met the ASAS 20 definition of response at one year. Non-responders were defined as those patients who did not meet the ASAS 20 definition of response at one year. Boxes represent the 25th and 75th centiles; horizontal lines represent the medians; vertical lines above and below the boxes represent the distribution beyond the 25-75th centile range. $p$ Value $<0.01$ for baseline CRP values. ) Comparison of TNF $\alpha$ levels in the supernatants of stimulated PBMC between responders and non-responders to infliximab in patients with AS. Responders were defined as those patients who met the ASAS 20 definition of response at one year. Non-responders were defined as those patients who did not meet the ASAS 20 definition of response at one year. See "Methods" for further definition of ASAS criteria for response. Boxes represent the 25 th and 75 th centiles; horizontal lines represent the medians; vertical lines above and below the boxes represent the distribution beyond the 25-75th centile range. $p<0.006$ for baseline TNF $\alpha$ values.

with even lower correlations detected when the cohort was classified into responders and non-responders.

\section{Cytokine analysis}

Baseline cytokine levels

Baseline TNF $\alpha$ levels were higher for responders than for non-responders $(p<0.006)$, with the responders having a mean (SD) level of $4797.8(2793) \mathrm{pg} / \mathrm{ml}$ ) as compared with (2607 (546) $\mathrm{pg} / \mathrm{ml}$ ) for non-responders (fig 1B). This difference was maintained at the 22 week assessment point $(\mathrm{p}<0.021)$. Apart from $\mathrm{TNF} \alpha$, there was no difference between the groups for the remaining baseline cytokine levels.

When response was defined using the ASAS 50 cut off point, there were 12 responders and 10 non-responders at year 1. Although no significant differences in baseline 
cytokine levels were noted between the groups, responders did have higher baseline TNF $\alpha$ levels (5014 (2805) pg/ml) than non-responders (3662 (2422) pg/ml).

\section{Correlation of clinical and immunological parameters with baseline cytokines}

Baseline BASDAI correlated poorly with baseline TNF $\alpha$ $\left(r_{\mathrm{s}}=0.09\right)$ for the entire cohort, and for responders $\left(r_{\mathrm{s}}=0.12\right)$ and non-responders $\left(r_{\mathrm{s}}=0.10\right)$. Similarly, a poor correlation was noted between baseline levels of CRP and the candidate cytokines, for the entire cohort and for responders (figs $2 \mathrm{~A}$ and $\mathrm{B}$ ). A strong correlation was noted between baseline CRP and ILl, IL10, and IFN $\gamma$ levels for nonresponders, suggesting that the profile of a non-responder is low baseline CRP, high ILl, high IL10, and low IFN $\gamma$ levels.

\section{Sequential cytokines}

When repeated measures ANOVA testing was used no significant differences were noted between the two groups of patients over the study period at the 0.05 level of significance.

However, a difference by $\chi^{2}$ analysis between responders and non-responders was noted after the first infusion (in the 0-2 week interval) in TGF $\beta$ and IL10 levels. During this interval the responders were differentiated from nonresponders by higher TGF $\beta$ levels and lower IL10 levels. Furthermore, the change in TGF $\beta$ and IL10 levels correlated highly with the change in BASDAI but not with CRP. Apart from these observations there was no relationship between response status and change in any of the remaining candidate cytokines over the specified time intervals.

\section{DISCUSSION}

In this study we found that patients who respond to infliximab as defined by the ASAS group criteria have higher levels of TNF $\alpha$ and higher CRP levels before treatment than non-responders. The other cytokines studied at baselinenamely, ILl, IL10, IFN $\gamma$, and TGF $\beta$, did not differ significantly between responders and non-responders.

The pathogenesis of AS is unexplained and as yet a signature cytokine profile has not been defined in this patient population. TNF $\alpha$ represents a strong candidate as a critical cytokine in the pathogenesis of this disease. Polymorphisms of the TNF $\alpha$ promoter gene have been reported by some groups, ${ }^{10}$ and local TNF $\alpha$ production has been demonstrated in the biopsies of inflamed sacroiliac joints. ${ }^{11}$ It is evident from short term trials that therapeutic blockade of TNF $\alpha$ is effective in most patients. But there are clearly patients who do not respond or who fail to sustain their initial response. Our findings suggest that baseline TNF $\alpha$ levels may be an important marker of this effect. On the basis of these findings we suggest that those patients in whom $\mathrm{TNF} \alpha$ is lower at baseline the spinal inflammation may not be as TNF $\alpha$ dependent as it is in their counterparts with higher TNF $\alpha$ levels, and that differential response to TNF $\alpha$ blockade is the consequence. This may be explained by the duration of disease in patients where those with longer disease duration have less TNF $\alpha$ dependent spinal inflammation. This hypothesis merits further study with larger numbers of patients as it has important implications for earlier intervention with a TNF $\alpha$ blocker in AS.

Two recent reports have examined changes in cytokines after infliximab treatment in spondyloarthropathy $(\mathrm{SpA}) .{ }^{4}$ In the first study the cohort was more heterogeneous as the inclusion criteria adopted the European Spondylarthropathy Study Group $(E S S G)^{12}$ classification of SpA, and cytokines were examined after only short term treatment with infliximab (three infusions). These authors demonstrated an increase in IFN $\gamma$ after treatment and a decrease in ILI0. We did not observe a comparable change in IFN $\gamma$, but there were differences in methodology. However, we did notice that there was a difference in IL10, with lower levels in responders than in non-responders after the first infusion. It was also noted in that study that before treatment patients exhibited an impaired Thl cytokine pattern that was restored after treatment with infliximab. In our study we used each patient as his own control, where the reference point for each treatment interval was each patient's respective baseline value.

In the second report, 20 patients with AS were selected from a cohort of 70 who participated in a randomised controlled trial..$^{5}$ Ten of these patients received infliximab for 12 weeks and 10 received placebo for six weeks and were then crossed over to infliximab treatment and evaluated after a further six weeks. The methodology differed from our study in several respects, which obviously makes any comparison between the studies difficult.

Zou et al demonstrated in this study that IFN $\gamma$ and TNF $\alpha$ secretion from $\mathrm{T}$ cells was down regulated significantly by six weeks and this was maintained at 12 weeks after the start of infliximab in the treatment group. In the placebo group no reduction in these cytokines was noted at six weeks, but on cross over to treatment both cytokines decreased significantly at six weeks. Baseline characteristics between groups, including disease activity, were not reported. This may be an important issue as baseline TNF $\alpha$ and IFN $\gamma$, seemed to be lower in placebo than in control groups. Possibly, the lack of reduction in TNF $\alpha$ and IFN $\gamma$ in the patients in the placebo group was due to a floor effect, whereby those patients had significantly lower values than the treatment group. Interestingly, similar to our findings, no significant pattern in sequential cytokine over 12 weeks was detected when evaluation was carried out on peripheral blood monocytes in the patients treated with infliximab.

Neither of the above studies stratified patients according to response to treatment so we do not know whether there were differences in baseline $\mathrm{TNF} \alpha$ secretion between responders and non-responders similar to those we found.

In SpA there is no inflammatory marker, such as CRP, that can reliably monitor disease activity. ${ }^{13}$ Our results are in

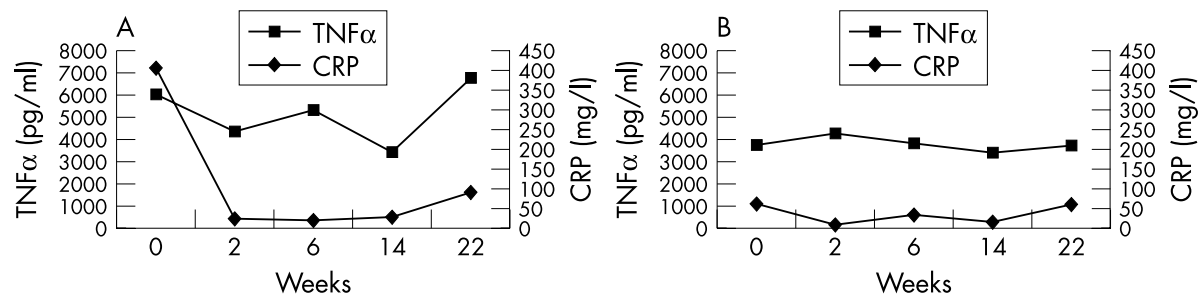

Figure 2 (A) A comparison of TNF $\alpha$ and CRP levels over the first six months of treatment with infliximab in responders. Response to treatment was defined using the ASAS 20 response criteria as before. (B) A comparison of TNF $\alpha$ and CRP levels in non-responders over the first six months of treatment with infliximab. Response to treatment was defined using the ASAS 20 response criteria as before. 
keeping with this observation, and baseline CRP levels correlated poorly with baseline disease activity. However, the wide variation in CRP levels among the subjects in this cohort might have contributed to the lack of a statistically significant difference. Interestingly, we found that baseline CRP levels were higher in responders than in nonresponders. Braun et al made a similar observation on their cohort. ${ }^{1}$ However, baseline levels of CRP did not correlate with TNF $\alpha$, which itself was the sole cytokine that distinguished responders from non-responders before treatment. This suggests that there may be an intermediate factor that is related to CRP and TNF $\alpha$ which may predict response also.

In conclusion, this study indicates that measuring CRP and $\mathrm{TNF} \alpha$ levels at baseline may be useful in identifying response patterns to infliximab in patients with AS before treatment. On the other hand, sequential cytokine analysis of PBMC does not appear to be informative. Further studies with larger numbers of patients are necessary to perform a multiple logistic regression analysis in order to determine if these variables are true predictors of response to treatment with infliximab.

\section{Authors' affiliations}

M A Stone, C Pacheco-Tena, R D Inman, Department of Medicine and Division of Rheumatology, University of Toronto; Toronto Western Hospital Research Institute, Toronto, Ontario, Canada

U Payne, Toronto Western Hospital Research Institute, Toronto, Ontario, Canada

Correspondence to: Dr R D Inman, Arthritis Centre of Excellence, Toronto Western Hospital, 399 Bathurst St, Toronto, Canada M5T 2S8; robert.inman@uhn.on.ca

Accepted 28 April 2003

\section{REFERENCES}

1 Braun J, Brandt J, Listing J, Zink A, Alten R, Golder W, et al. Treatment of active ankylosing spondylitis with infliximab: a randomized controlled multicentre trial. Lancet 2002;359:1187-93.

2 Gorman JD, Sack KE, Davis JC Jr. Treatment of ankylosing spondylitis by inhibition of tumor necrosis factor alpha. N Engl J Med 2002;346:1349-56.

3 Van Den Bosch F, Kruithof E, Baeten D, Herssens A, De Keyser F, Mielants H. Randomized double-blind comparison of chimeric monoclonal antibody to tumor necrosis factor alpha (infliximab) versus placebo in active spondylarthropathy. Arthritis Rheum 2002;46:755-65.

4 Baeten D, Van Damme N, Van Den Bosch F, Kruithof E, De Vos M, Mielants H, et al. Impaired Th1 cytokine production in spondyloarthropathy is restored by anti-TNF alpha. Ann Rheum Dis 2001;60:750-5.

5 Zou JX, Rudwaleit M, Brandt J, Thiel A, Braun J, Sieper J. Down-regulation of the nonspecific and antigen-specific T cell cytokine response in ankylosing spondylitis during treatment with infliximab. Arthritis Rheum 2003;48:780-90.

6 van der Linden S, Valkenburg HA, Cats A. Evaluation of diagnostic criteria for ankylosing spondylitis: a proposal for modification of the New York criteria. Arthritis Rheum 1984;27:361-8.

7 Stone M, Salonen D, Lax M, Payne U, Lapp V, Inman R. Clinical and imaging correlates of response to treatment with infliximab in patients with ankylosing spondylitis. J Rheumatol 2001;28:1605-14.

8 Calin A, Garrett S, Whitelock H, Kennedy G, O'Hea J, Mallorie P, et al. A new approach to defining functional ability in ankylosing spondylitis: the development of the Bath Ankylosing Spondyltis Functional Index. J Rheumatol 1994;21:2281-5.

9 Anderson JJ, Baron G, van der Heijde D, Felson DT, Dougados M. Ankylosing spondylitis assessment group preliminary definition of short-term improvement in ankylosing spondylitis. Arthritis Rheum 2001;44:1876-86.

10 Stone MA, Inman RD. The genetics of cytokines in ankylosing spondylitis. J Rheumatol 2001;28:1203-6.

11 Braun J, Bollow M, Neure L, Sieptelt E, Seyerekbasan F, Herbst H. Use of immunohistologic and in situ hybridization techniques in the examination of sacroiliac joint biopsy specimens from patients with ankylosing spondylitis. Arthritis Rheum 1995;38:499-505.

12 Dougados M, van der Linden S, Juhlin R, Huiffeldt B, Amor B, Calin A. The European Spondyloarthropathy Study Group preliminary criteria for the classification of spondyloarthropathy. Arthritis Rheum 1991;34:1218-27.

13 Spoorenberg A, van der Heijde D, de Klerk E, Dougados M, de Vlam K, Mielants $\mathrm{H}$, et al. Relative value of erythrocyte sedimentation rate and $\mathrm{C}$-reactive protein in assessment of disease activity in ankylosing spondylitis. J Rheumatol 1999;26:980-4. 